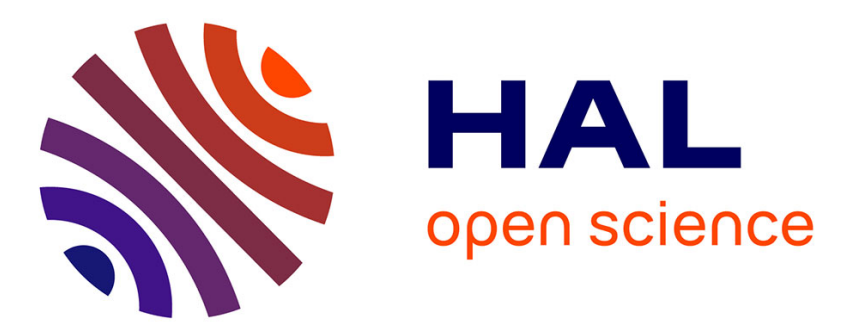

\title{
Dual-primal skeleton: a thinning scheme for vertex sets lying on a surface mesh
}

Ricardo Uribe Lobello, Jean-Luc Mari

\section{To cite this version:}

Ricardo Uribe Lobello, Jean-Luc Mari. Dual-primal skeleton: a thinning scheme for vertex sets lying on a surface mesh. 14th International Symposium on Mathematical Morphology (ISMM 2019), Jul 2019, Saarbrücken, Germany. 10.1007/978-3-030-20867-7_6 . hal-02130490

\section{HAL Id: hal-02130490 \\ https://hal.science/hal-02130490}

Submitted on 23 May 2020

HAL is a multi-disciplinary open access archive for the deposit and dissemination of scientific research documents, whether they are published or not. The documents may come from teaching and research institutions in France or abroad, or from public or private research centers.
L'archive ouverte pluridisciplinaire HAL, est destinée au dépôt et à la diffusion de documents scientifiques de niveau recherche, publiés ou non, émanant des établissements d'enseignement et de recherche français ou étrangers, des laboratoires publics ou privés. 


\title{
Dual-primal skeleton: a thinning scheme for vertex sets lying on a surface mesh
}

\author{
Ricardo Uribe Lobello* and Jean-Luc Mari \\ Aix Marseille Université, Université de Toulon, CNRS, LIS, Marseille, France
}

\begin{abstract}
We present a new algorithm for the skeletonization of shapes lying on surface meshes, which is based on a thinning scheme with a granularity that is twice as fine as that of other thinning methods, since the proposed approach uses dual-primal iterations in the region of interest to perform the skeleton extraction. This dual operator is built on specific construction rules, and it is applied until idempotency, which provides a better geometric positioning of the skeleton compared to other thinning methods. Moreover, the skeleton has the property of ensuring the same topological guarantees as other homotopic thinning approaches: the skeleton is thin, connected and can include Y-branches and cycles if the input region contains holes.

Keywords: skeletonization, surface mesh, homotopic thinning, shape description, topology preservation.
\end{abstract}

\section{Introduction}

\subsection{Context, problem and related work}

The skeleton is a well-known shape descriptor. It is an entity that is globally centered in a 2D or 3D object, and it characterizes its topology and its geometry. This structure is widely used in various applications (video tracking [4, shape recognition [13, surface sketching [9], etc.). Several techniques exist in order to extract the skeleton from binary 2D images [14, 3D closed volume meshes [2, or $3 \mathrm{D}$ cubic grids 8 .

Nevertheless, very few approaches have been dedicated to the extraction of skeletons from binary information located on an arbitrary triangulated mesh (see [3]12]11] for the state of the art). Therefore, the task of computing the skeleton of the subset of a discrete surface embedded in $\mathbb{R}^{3}$ remains. Rössl et al. 10] have presented the first method that uses the elementary mathematical morphology opening operator, ported to triangulated meshes. However, the operator's definition is incomplete, and the underlying algorithm presents some issues. Therefore, several drawbacks have been pointed out, which mainly lead to unexpectedly disconnected skeletons 5 . Kudelski et al. have later proposed a modified algorithm that produces topologically robust skeletons by generalizing the notion of morphological erosion to arbitrary meshes [7]6]. This approach takes, as an input, a subset lying on a triangulated surface mesh in $3 \mathrm{D}$, and as 
outputs, thin lines corresponding to the skeleton obtained by homotopic thinning. The main idea is to transpose the notion of neighborhood from the classical thinning algorithms where the adjacency is constant (e.g., 26-adjacency in digital volumes, 8 -adjacency in $2 \mathrm{D}$ grids) to the mesh domain where the neighborhood is variable due to the adjacency of each vertex.

\subsection{Contribution}

Our work continues the idea of homotopic thinning using a generalized adjacency described in [6]. Instead of iteratively removing nonrelevant vertices of the subset (topologically speaking, i.e., the simple vertices), the erosion step is replaced by a dual-primal operation. The area of interest is converted to the dual space, and all semi-infinite edges are removed from the structure. This process is repeated (dual to primal to dual) until idempotency. It produces a lineal skeleton with a resolution increased by a factor 2 , as the resulting structure is not only composed of initial vertices and edges but also composed of dual vertices and edges. This skeletonization is more general because it can address nondevelopable surfaces (the operations are local, and there is no need to have a $[i, j]$ indexing such as in $2 \mathrm{D}$ grids). Moreover, the resulting skeleton preserves the topology of the original shape lying on the surface.

This paper is divided as follows: section 2 briefly develops some basic notions and definitions. Then, section 3 explains in detail our approach of dual-primal skeletonization using a specific thinning scheme on surface meshes. Section 4 is dedicated to the validation of the method, including tests on irregular meshes and an application to the extraction of feature lines.

\section{Basic notions}

Let $\mathcal{M}$ be an unstructured mesh patch representing an arbitrary manifold surface $\mathcal{S}$, such as $\mathcal{M}=(\mathcal{V}, \mathcal{E}, \mathcal{T})$. The sets $\mathcal{V}, \mathcal{E}$ and $\mathcal{T}$ correspond, respectively, to the vertices, the edges, and the triangles composing $\mathcal{M}$, a piecewise linear approximation of $\mathcal{S}$. We denote $p_{i}$ the vertices, with $i \in[0 ; n[$ and $n=|\mathcal{V}|$ being the number of vertices in $\mathcal{M}$. The neighborhood $\mathcal{N}$ of a vertex $p_{i}$ is defined as follows:

$$
\mathcal{N}\left(p_{i}\right)=\left\{q_{j} \mid \exists\left(p_{i}, q_{j}\right) \in \mathcal{E}\right\} .
$$

In such a case, $m_{i}=\left|\mathcal{N}\left(p_{i}\right)\right|$ represents the total number of neighbors of $p_{i}$. As we consider obtaining a skeleton of a subset of $\mathcal{M}$, let us now define a binary attribute $F$ on each vertex of $\mathcal{V}$. The set $R \subseteq \mathcal{V}$ is then written as follows:

$$
\forall p_{i} \in R \Longleftrightarrow F\left(p_{i}\right)=1 .
$$

The attribute $F$ may be defined from a previous process such as manual selection, thresholding based on geometric properties (triangle area, principal curvatures, etc.) or any binarization process. Then, an edge $e=(p, q)$ belongs to $R$ if and only if $p, q \in R$. Similarly, a triangle $t=(p, q, r)$ belongs to $R$ if and only if $p, q, r \in R$. 


\section{Dual-primal skeletonization}

Our approach is based on a robust dual-primal erosion algorithm that preserves the topology of the original mesh. In the next sections, we present an overview of our algorithm and a detailed explanation of each part of our algorithm.

\subsection{Overview of the approach}

The input to our algorithm can be any triangular mesh $\mathcal{M}$ that is a set of triangular faces connected by edges and vertices as defined in section 2. As in section 2 , this mesh must have the vertices marked with a function in order to define regions of interest. $\mathcal{M}$ can contain several connected components, and it can have one or several borders. Hereafter, we will call the input mesh the initial primal mesh (IPM). Our approach is straightforward; it starts by extracting the dual representation of the IPM. Then, it detects the faces that can be eliminated by exploring the dual mesh. Finally, it computes the intermediate primal representation of the mesh by eliminating the primal faces that are not going to affect the topology of the final skeleton. Then, we repeat the dual-primal iteration over this new primal mesh. Finally, it stops when the primal mesh in iteration $N$ is equal to the primal mesh in iteration $N+1$. The main steps of our approach are illustrated in figure 1.

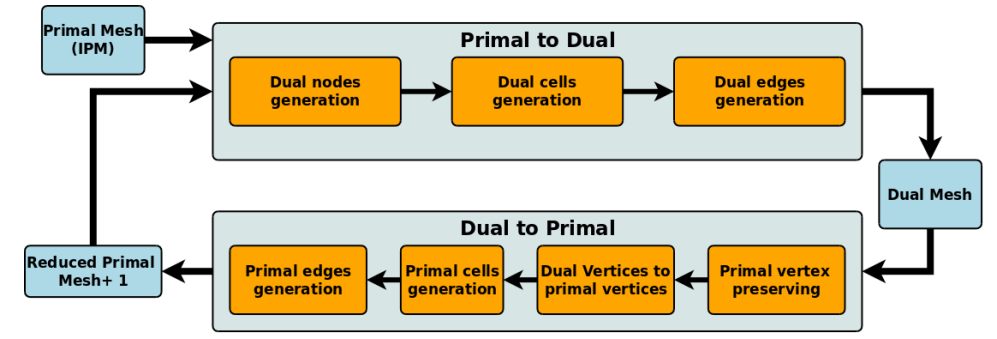

Fig. 1: General structure of the workflow of our approach. The initial mesh is provided as input. A dual representation is computed. Then, a primal mesh is obtained by eliminating those dual cells that are not totally contained in the areas of interest. The new primal mesh can contain edges representing the thin features of the input mesh. Finally, the reduced mesh is provided as input to the next iteration until idempotency.

The primal-to-dual and dual-to-primal transformations are performed by following a well-defined set of rules that guarantee that the final mesh will preserve the topology of the IPM.

\subsection{Primal-Dual-Primal computation}

In classic dual extraction algorithms, the dual mesh is extracted based on an edge adjacency relationship between cells. For each edge in the primal mesh, 
a dual edge is generated. However, this narrow definition is not sufficient to obtain a 1-dimensional skeleton because it does not consider vertex adjacencies between edges and vertex adjacency between faces. Vertex adjacency is necessary to detect all connected components in the area of interest in the original mesh. Furthermore, as our algorithm applies an erosion to the input mesh, it will eventually lead to the creation of mixed meshes containing 2-dimensional (faces) and 1-dimensional (edges) cells that are uniquely connected by vertices. Our approach addresses these cases in order to keep the consistency and fidelity of the final mesh with respect to the IPM.

\subsection{General rules for dual mesh generation}

These rules follow classic definitions of dual meshes extracted from polygonal meshes.

1. Each primal cell is replaced by a dual vertex. It includes primal faces and primal edges. Initially, we always place the dual vertex in the barycenter of the primal cell as illustrated in figures $2 \mathrm{a}$ and $2 \mathrm{~b}$.

2. Each primal vertex is replaced by a dual cell. In the case of vertices surrounded by 2-dimensional cells, the dual cell is a polygon. In the case of a vertex surrounded by edges, it is replaced by a dual edge. These cases are illustrated in figures 2 and $2 \mathrm{~d}$.

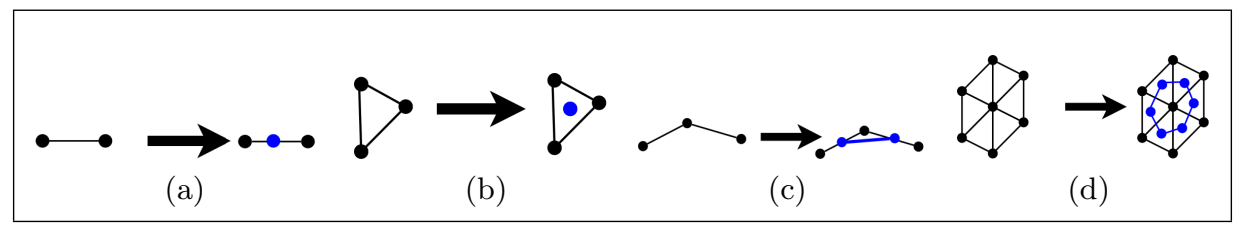

Fig. 2: Basic transformations from a primal mesh to a dual mesh. (a) Primal edge to dual vertex. (b) Primal face to dual vertex. (c) Primal vertex to dual edge. (d) Primal vertex to dual cell (a polygon).

As a consequence of the previous rules, starting from a primal mesh $\mathcal{M}_{k}=$ $\{\mathcal{V}, \mathcal{E}, \mathcal{C}\}$, its dual mesh $\mathcal{D}_{k}=\left\{\mathcal{V}_{d}, \mathcal{E}_{d}, \mathcal{C}_{d}\right\}$ (in the general case) is defined by equations:

$$
\begin{gathered}
\mathcal{V}_{d}=\left\{v_{d_{i}} \mid \exists c_{i} \in \mathcal{C} \text { so that } v_{d_{i}} \text { is located at the barycenter of } f_{i}\right\} \\
\qquad \begin{array}{c}
\mathcal{E}_{d}=\left\{e_{d_{i}} \mid \exists e_{i} \in \mathcal{E}\right\} \\
\mathcal{C}_{d}=\left\{c_{d_{i}} \mid \exists v_{i} \in \mathcal{V}\right\}
\end{array}
\end{gathered}
$$

These definitions apply, as mentioned, in the general case of a closed mesh without borders. The IPM can have borders, and thus, the intermediate meshes 
can contain borders. Therefore, the previous definitions have to be expanded to consider these special cases. For example, the region $\mathcal{R}$ can contain thin structures; hence, it is not possible to generate the classic dual cell, but it is possible to generate linear dual cells. We will explain these cases in more detail in the next section.

\subsection{Generation of dual cells in particular cases}

To address all possible cases in a region with borders and to prove its topological correctness, it is necessary to expand our definition of a dual mesh.

Dual vertex generation Our algorithm simply traverses each cell in the primal mesh $\mathcal{M}$ and computes a dual vertex placed in the barycenter of the current cell. This algorithm is easily applied over 1-dimensional and 2-dimensional cells. There is no need to extend the current definitions.

Dual cell generation In the general case, it is possible to build the dual cell $d_{i}$ around a primal vertex $c_{i}$ by extracting its face neighborhood as defined in equation 6

$$
\mathcal{N}_{f}\left(c_{i}\right)=\left\{f_{j} \mid c_{i} \in \mathcal{V}\left(f_{j}\right) \text { where } \mathcal{V}\left(f_{j}\right) \text { is the set of vertices of cell } f_{j}\right\} .
$$

From $\mathcal{N}_{f}\left(c_{i}\right)$, it is possible to generate the dual vertices that will be connected to generate the dual cell. However, it is necessary to ensure that the faces are ordered counterclockwise in order to obtain a well oriented dual mesh. To do this, our algorithm extracts the ordered neighborhood of the primal vertices around $c_{i}$ by traversing all faces incidents to $c_{i}$. Then, we extract all edges in those faces not containing $c_{i}$. Finally, we order these edges to create a closed path. This algorithm only works in well oriented surfaces. Thus, this vertex neighborhood can be redefined as follows.

$$
\mathcal{N}_{v}\left(c_{i}\right)=\left\{c_{j} \in \mathcal{V}\left(f_{k}\right) \mid \exists f_{k} \in \mathcal{N}_{f}\left(c_{i}\right) \text { and } c_{j} \neq c_{i}\right\} .
$$

The vertices contained in $\mathcal{N}_{v}\left(c_{i}\right)$ can be ordered to check if the current vertex $c_{i}$ is actually surrounded by a closed path of vertices. If it is the case, it is possible to generate the dual cell. If it is not the case, the current vertex belongs to a thin structure or a border. Our algorithm to process these cases will be explained in the next section.

It is necessary to also handle the cases where 1-dimensional and 2-dimensional cells are adjacent. In these cases, it is necessary to make a decision about the way how these cells have to be connected, which occurs in both the primal space and in the dual space, and the method used to solve this is presented in the next section.

\subsection{Handling mixed regions}

Mixed regions usually appear when large components encounter thin structures. It can happen in the transition from primal to dual and in the dual-to-primal transformation. Therefore, we develop these two transitions separately. 
Primal to Dual Transformation Once the dual vertices have been generated, it is necessary to detect thin structures to avoid the separation of components connected by a strip of triangles or a set of vertices. To detect if a primal face $f$ is contained in a thin structure, we traverse each of its primal vertices checking if a ring can be built around each one. If this is not possible for any of them, this primal face is marked as comprising part of a thin structure. Then, each dual vertex in every face is connected to the dual vertex in the edge-adjacent cell in order to generate a dual edge. This process is repeated until all dual vertices in the thin structure are connected. It is important to clarify that edges generated in thin structures are 1-dimensional dual cells and are stored explicitly, in contrast to edges comprising part of 2-dimensional dual cells, which are stored implicitly. This process is illustrated in figure 3 .

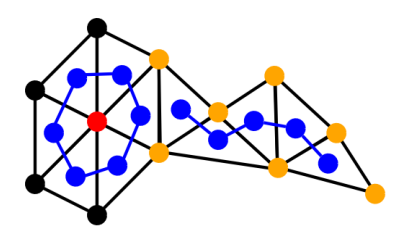

Fig. 3: Detection of thin structures. For each primal vertex, we check the 1-ring built from its incident faces. In red, a primal vertex in a larger structure. In yellow, these primal vertices do not have a 1-ring around them. Consequently, all primal faces containing only these kinds of vertices are part of a thin structure. In blue are dual vertices, dual faces and dual edges.

As seen in figure 3, dual vertices in thin structures are still not connected to dual vertices in the larger structure. Our algorithm traverses all dual vertices belonging to thin structures to detect end points, which is done by checking if the dual vertex is only adjacent to one 1-dimensional cell. Then, for each end point $v_{\text {endpoint }}$, we obtain its primal cell $c_{p}$, and we check if $c_{p}$ is an edge-adjacent to another primal cell $c_{a}$ so that it contains a dual vertex belonging to a 2dimensional dual cell. We do this only once in order to avoid connecting the thin structure with the larger structure multiple times. This procedure is listed in the algorithm 1 .

As mentioned previously, our algorithm connects vertex-adjacent regions in order to preserve the topology of a region of interest defined with marked vertices, which is implemented in the primal-to-dual phase. Dual vertices in vertexadjacent primal vertices are always connected by an edge. It is important to remember that a primal cell can be edge-adjacent to several structures. Consequently, it is necessary to detect all components that have to be connected with the current dual vertex, which is illustrated in figure 4 a.

To connect these structures, it is necessary to detect one of the end points $v_{a}$ or $v_{b}$. Then, the algorithm extracts the set of primal cells $C$ that are adjacent to the shared primal vertex $v$. Next, we extract the connected components of $C$. Finally, we choose one of the primal cells belonging to each connected component 
Algorithm 1: Connecting the dual vertices in a thin structure with the dual vertices in larger structures.

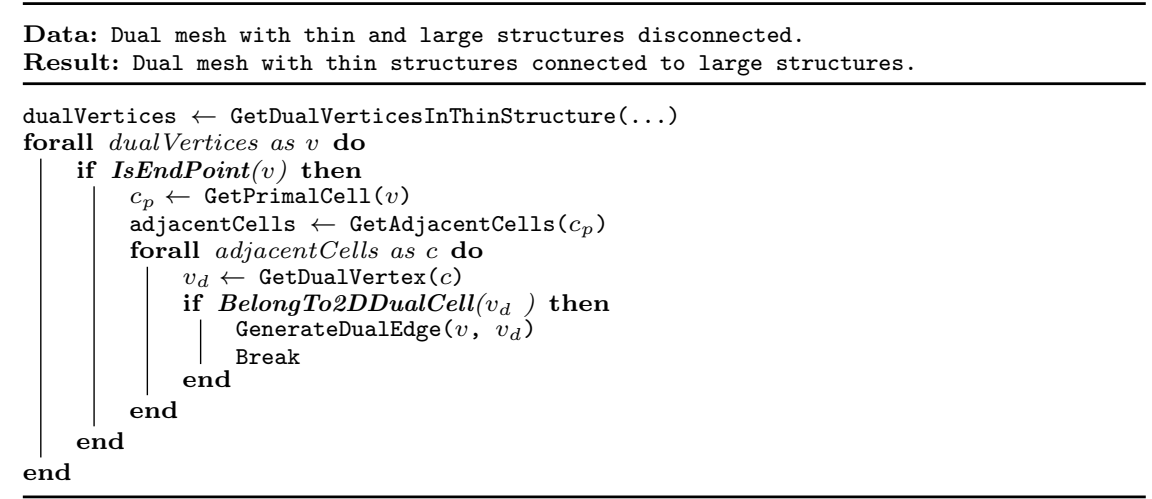

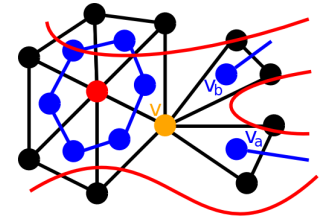

(a)

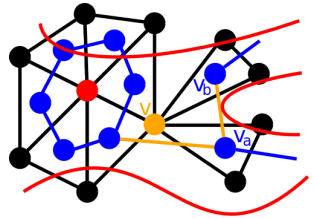

(b)

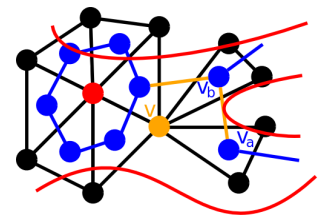

(c)

Fig. 4: In red, we illustrate potential borders for the structure approximated by the area of interest. (a) Vertex-adjacent components in the Dual cells and dual vertices are in blue. The large structure is composed of a dual cell in blue. This structure is connected to two thin structures by the primal vertex in orange. (b) The three components are connected by the orange edges from $v_{a}$. It is clear, however, that we can obtain different meshes depending on the initial dual vertex (c). As explained later, we do not consider this as a limitation for our approach because the topology of the area of interest is preserved.

that contains a dual vertex, and we connect $v_{a}$ (or $v_{b}$ ) with each one of these dual vertices. This procedure is listed in algorithm 2 and illustrated in figure $4 \mathrm{~b}$.

By using this method, it is possible to obtain different meshes from the same input depending on the dual vertex that is first detected $\left(v_{a}\right.$ or $\left.v_{b}\right)$. However, as this choice does not change the topology (but the connectivity) of the final mesh. As our objective is to preserve the topology in the sense of connected components, holes and voids and not in terms of connectivity of the mesh, this result is correct.

Dual-to-Primal Transformation Once a dual mesh has been generated, our approach detects which primal cells can be eliminated without affecting the topology of the final linear structure. To do this, we apply the following rule: 
Algorithm 2: Connecting the dual vertices of vertex-adjacent primal cells. This algorithm can connect multiple dual vertices from multiple vertexadjacent primal cells (see figure 4).

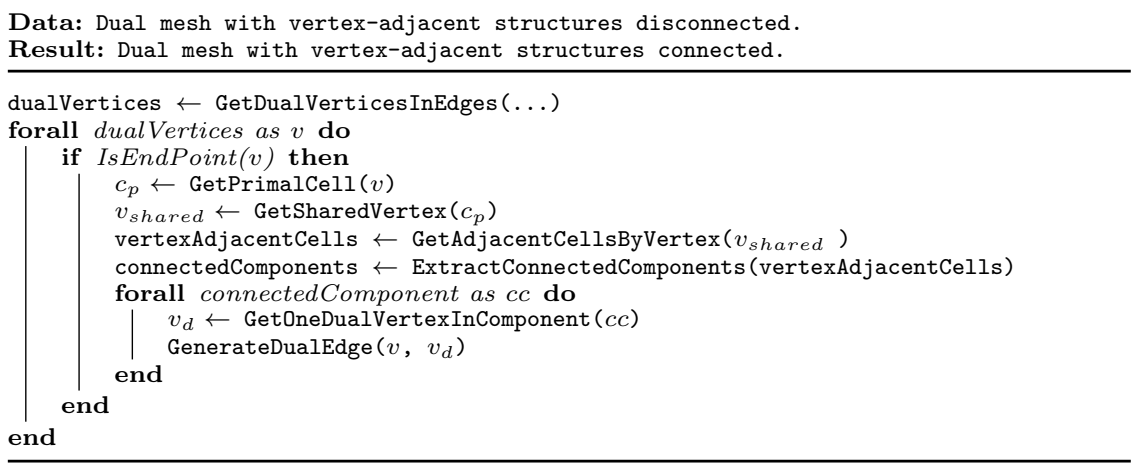

Rule 1 A primal cell must be preserved if and only if its dual vertex is shared by three $2 D$ dual cells.

The rule 1 is intended to preserve primal cells that are part of large structures. This rule also implies that all primal cells corresponding to a dual vertex shared uniquely by one or two $2 \mathrm{D}$ dual cells must be eliminated. These primal cells are, evidently, always located in the frontiers of the structure. Therefore, the application of rule 1 results in an erosion of the larger structures from the border to the inside, similar to the prairie fire algorithm used in topological thinning methods. It is implemented by traversing all dual vertices and obtaining its adjacent dual cells.

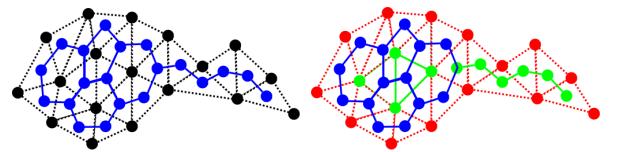

(a) (b)

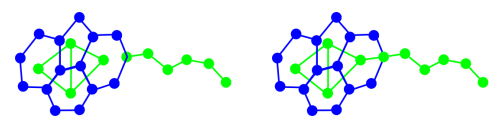

(c) (d)

Fig. 5: General illustration of the transition from dual to primal. This example shows a large structure connected to a thin structure.

The rule 1 is only intended to preserve interior faces. The connectivity between large and thin structures is represented as a dual edge sharing a dual vertex with a 2-dimensional dual cell, as explained in the previous section. To preserve this topology in the next primal mesh, we proceed as follows. In the case of primal faces belonging to thin structures and, as consequence, represented in the dual mesh by dual vertices, they are not preserved; however, each one is replaced by its dual vertex, which is added to the next primal mesh. Dual edges inside these thin structures are all preserved as primal edges. Finally, dual edges connecting to 2-dimensional dual cells are also preserved by adding the shared 
dual vertex to the primal vertices and connecting it to the primal vertex at the origin of the primal 2-dimensional cell.

This process is illustrated in figure 5 with the original mesh in black and the initial dual mesh in blue. In figure 5 b, green faces and vertices are preserved, all primal faces and vertices that are going to be eliminated (by using the complement of rule 1) are in red. In 5e, thin and large structure are still not connected. In 5d, These structures are connected by using the primal vertex at the origin of the 2-dimensional dual cell and the dual vertex in the same dual cell connected to the thin structure.

The method previously described is sufficiently general to address several thin structures or branches converging toward a single 2-dimensional dual cell and can generate multiple junctions at the same point; it preserves the topology of the original area of interest.

As evident from the primal mesh obtained in figure $5 \mathrm{~d}$, our approach generates intermediary meshes that are a mixture of faces and edges as illustrated in figure 6a. Therefore, this kind of configuration has to be addressed in the primal-todual transition. In these cases, the algorithm connects the dual vertex in the primal edge with the dual vertex belonging to the adjacent primal face, forming a junction as shown in figure $6 \mathrm{~b}$.

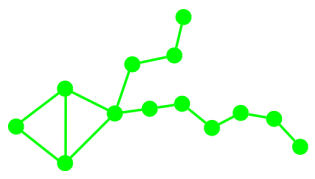

(a)

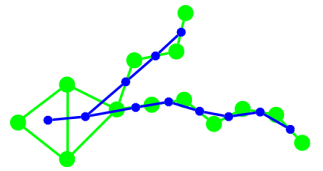

(b)

Fig. 6: General illustration of the extraction of a dual mesh in areas of the mesh where primal faces and edges are connected. (a) This primal mesh has been generated from a previous iteration. It generates mixed meshes with edges and faces. (b) The dual mesh in blue is generated mostly by using the standard rules explained earlier. However, in mixed areas, dual vertices in edges have to be connected to dual vertices in primal faces generating a junction.

The previous primal-to-dual and dual-to-primal processes are applied iteratively until idempotency. In this final stage, we obtain a mesh containing edges only. These edges are positioned approximately at the center of the topological structures of the original area of interest. This fact will help to better understand the next section.

\subsection{Geometric positioning}

The algorithm generates linear meshes well positioned in the center of the main structures of the original object, which is possible because of the symmetrical erosion procedure that is used to progressively eliminate the most external primal faces in the mesh. As explained above, our algorithm advances from the borders of the mesh to the interior. Thus, the final structure converges slowly towards the center of the structure. Additionally, we use the primal vertices if possible; 
however, if necessary, we generate and add dual vertices to the next primal mesh. These dual vertices are placed in the barycenter of primal faces, thus increasing the centering of the final structure, especially in thin structures.

Concerning the geometric position with respect to the original surface, we use, if possible, the original primal vertices. If dual vertices are used in the final mesh, they are strictly located over the primal faces. Therefore, the vertices of the final mesh are always located over the original mesh. By contrast, final edges are not always located on the surface. It is only the case when the edge is relying two initial primal meshes. In any other case, all the points of the edge are not necessarily on the surface.

\subsection{Topological guarantees}

The topological guarantees offered by our approach can be proven by analyzing the different cases that can appear in the primal-to-dual and dual-to-primal transitions.

Lemma 1. In the transition from the primal mesh to the dual mesh, all topological structures are represented through their dual counterparts.

Proof. Let $A_{p}=\left\{V_{p}, F_{p}, E_{p}\right\}$ with $V_{p}$ be a set of primal vertices, $F_{p}$ be a set of primal faces and $E_{p}$ be a set of edges. We consider $\mathcal{S}_{p}$ a conformal mesh so that N-dimensional cells only intersect on (N-1)-dimensional cells. Triangles only intersect in an edge or a vertex and edges only intersect in a vertex. Therefore, we consider that $A_{p}$ is also conformal.

First, let the dual mesh be defined equivalently as $A_{d}=\left\{\mathcal{V}_{d}, \mathcal{F}_{d}, \mathcal{E}_{d}\right\}$. As our algorithm generates one dual vertex for each primal cell or edge, we find that the set of dual vertices $\mathcal{V}_{d}$ is composed of dual vertices centered at their corresponding primal cell, as illustrated in figure 2 and 2 o. Hence, each face $f_{p}^{i} \in F_{p}$ or edge $e_{p}^{i} \in E_{p}$ in the region $A_{p}$ is represented by a dual vertex $v_{d}^{i} \in \mathcal{V}_{d}$.

Uniform regions : In the case of 2 edges connected by a primal vertex, dual vertices are connected by a dual edge (see 2 c). In the case of primal vertices surrounded by faces, dual vertices are connected as illustrated in figure $2 \mathrm{~d}$. This construction guarantees that linear structures will still be represented by linear (but dual) structures. In the case of triangulations, they will be represented by at least one dual cell.

Mixed regions : these are regions where a triangulation representing a large structure $L_{p}$ is connected to a thin structure $T_{p}$ by at least a primal vertex. These thin structures can be a strip of triangles or a set of edges. Two cases can be considered:

- If $L_{p} \cup T_{p}$ is an edge: The dual vertices belonging to the edge-adjacent triangles are connected by a dual edge, thus connecting the two regions.

- If $L_{p} \cup T_{p}$ is a primal vertex: $L_{p}$ and $T_{p}$ are either two primal faces or a primal face and a primal edge. Then, the dual vertex in $T_{p}$ is connected to the first primal cell of each vertex-adjacent connected component as illustrated in figure 4 . 
Mixed regions in the primal mesh only transform into dual edges in the dual mesh. As we have assumed that the area of interest is a conformal surface, the cases explained above connect all cells that are at least connected by a primal vertex. Consequently, this algorithm preserves the topology of the original surface.

Lemma 2. In the transition from the dual mesh to the primal mesh, all topological structures are preserved.

Proof. To prove lemma 2 we need to proceed by case:

- Dual edges: Dual edges are only converted to primal edges. Therefore, thin structures are preserved.

- Large structures: As mentioned previously, all internal dual vertices are transformed to primal cells. All noninternal dual vertices and their corresponding primal faces are eliminated. If no internal dual vertices exist, it means it is a thin structure; in any other case, primal faces will be conserved, and this structure will be present in the next iteration.

- Mixed structures: A 2-dimensional dual cell connected to a dual edge. In this case, the connection between the components is always conserved because the primal vertex at the center of the dual edge will be connected to the primal vertex at the origin of the 2-dimensional dual cell.

In conclusion, no components that have been connected during the primal-todual process are separated in the dual-to-primal transition. All connections are preserved in the edge and vertex adjacency. Consequently, the topology of the original region of interest is preserved.

\subsection{Post-processing}

Pruning Our algorithm uses a simple criterion to eliminate parasite branches. It consists of the detection of ending vertices of the structure. For each of these ending vertices, it checks if it belongs to the original mesh. If this is the case, this branch is preserved because it can represent an important feature of the region. If the ending vertex does not belong to the initial mesh, it means that it has been created during the primal-dual-primal iteration process. In this case, it is not sure that the vertex reflects a thin structure in the initial mesh. In this article, we have decided to keep these branches if they have a minimum length (a parameter) but more research is needed to establish if they really have to be conserved.

Smoothing As our approach is based entirely on the geometrical information of the mesh, it can produce highly oscillating structures. To improve these results, we apply a simple smoothing algorithm that moves every vertex to the barycenter of the vertices composing its topological neighborhood similar to the "Beatifying" algorithm proposed in [1.

Evidently, as this process can affect the approximation quality of the final skeleton, we bound the maximum approximation error from the original mesh. 


\section{Results and validation}

These results have been obtained in the Ubuntu 16.04 LTS 64-bit environment with the processor, Intel i7-7820HQ CPU @ $2.90 \mathrm{GHz} \times 8$ and 32 GBytes of internal memory. It has been implemented in $\mathrm{C}++$, and the mesh data structure used was the vtkPolyData. We used VTK built-in methods to traverse the mesh and access to its properties. Our approach is intended to work in 2-dimensional simplex meshes that can be mapped in 3D in order to represent volumes. For this, we have started applying it to $2 \mathrm{D}$ planar meshes and the results are discussed in the next section.

\subsection{Application on planar meshes}

In figure 7, our implementation is applied to four different meshes. These meshes are regular meshes with relatively well-formed faces. In the figure, we show the original mesh and the extracted skeleton after cleaning and some iterations of the smoothing process. Table 1 shows relevant information about the examples presented in figure 7 .

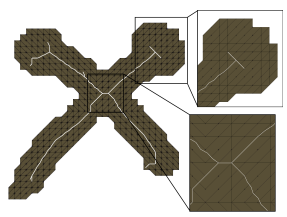

(a) Cross.

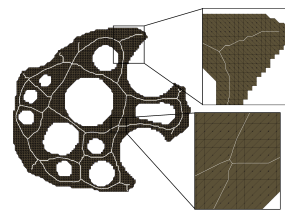

(b) Shape.

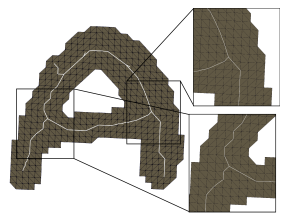

(c) Letter A.

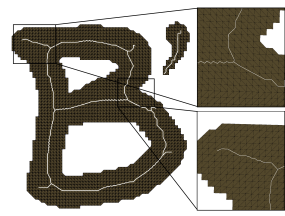

(d) Letter B.

Fig. 7: Application of our algorithm to 2D meshes. The cleaned skeleton is shown after a smoothing process.

Table 1: Information on the execution of the algorithm in the four previous planar meshes. This table shows the number of primal-to-dual and dual-to-primal iterations, the execution time and the total number of edges in the final skeleton.

\begin{tabular}{|c|c|c|c|c|}
\hline Data set & \# Triangles & \# Iterations & Exec. Time (in ms) & Lines Final skeleton \\
\hline Cross & 819 & 5 & 246 & 108 \\
\hline Shape & 8556 & 13 & 4529 & 1009 \\
\hline A letter & 812 & 6 & 261 & 103 \\
\hline B Letter & 4307 & 9 & 2049 & 361 \\
\hline
\end{tabular}

From figure 7, it is clear that the algorithm is able to extract skeletons from arbitrary triangular meshes while preserving the topology of the original mesh. It keeps junctions and holes, and places the final skeleton at approximately the center of the topological structure. However, the method is entirely based on the geometry and the connectivity of the mesh, and consequently, a different triangulation of the same object will produce a different skeleton.

Concerning the execution time, our implementation is not optimal because of the choice of the vtkPolyData structure. The vtkPolyData depends on a logarithmic data structure to traverse neighboring cells and adjacent vertices. Having 
into account the vtkPolyData data structure, the complexity of the dual vertex generation process is $\mathcal{O}\left(N \frac{1}{K}(F+V)\right)$ and including the complexity of the dual cell generation, the final complexity of our method is $\mathcal{O}\left(N\left[\frac{1}{K}[(F+V)+V \log F]\right)\right.$. As seen, the execution time of our algorithm is highly dependent of the size and connectivity of the input mesh. As optimization and with a half-edge data structure, the $\frac{1}{k} V \log F$ part of our approach can be optimized to a constant factor.

\subsection{Application on surfaces}

We have applied our approach to surfaces mapped in 3D that represent volumes. These surfaces have to be marked in order to define areas of interest. In figure 8. we show two surface meshes where areas of maximum curvature are marked. Our approach has extracted skeletons close to the center of interest areas.

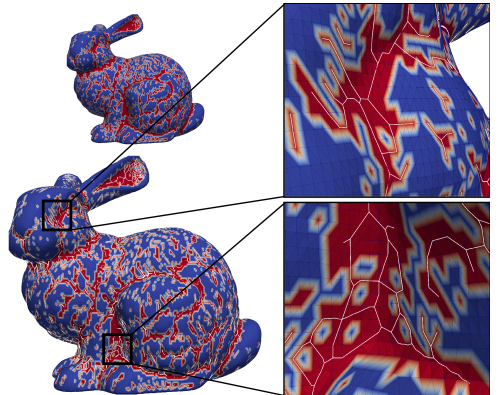

(a) Surface Bunny with 69451 faces and 35947 vertices. The final skeleton has 9053 edges.

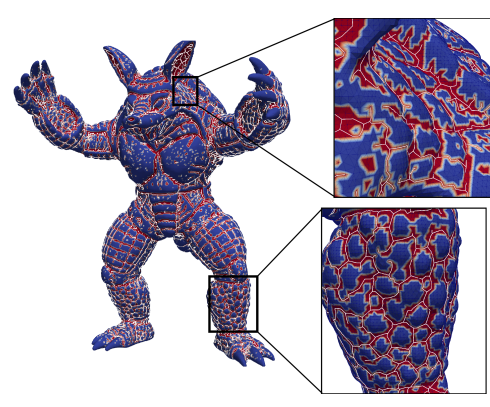

(b) Surface Armadillo with 345944 faces and 172974 vertices. The final skeleton has 46725 edges.

Fig. 8: Application to the high curvature areas of a surface (in red). Two closeups show how the skeleton (in white) is located in the middle of the region of interest.

As seen in figure 8, our algorithm is robust enough to be applied to any $2 \mathrm{D}$ mesh with complex topology. It is able to connect connected components with edge and vertex connectivity, allowing us to preserve the topology of thin structures connected only by 1-dimensional cells or edges. However, our current implementation does not guarantee that the skeleton is located in the center of the connected component but tends to place it close to it. To offer guarantees on the location of the skeleton, it will likely be necessary to follow closely the position of dual vertices, thus minimizing its distance to the center of mass of the current component. This kind of implementation can be used to extract the main features of surface meshes in the future.

\section{Conclusion and future work}

We have tested our approach and we have confirmed that the algorithm is robust and capable of extracting a 1-dimensional structure from any region lying on a mesh with arbitrary topology. However, this approach is highly dependent on 
the size and connectivity of the mesh. Thus, we think that a more efficient mesh data structure can strongly improve the complexity and the execution time of our method.

As future work, we consider that our approach is not limited to $2 \mathrm{D}$ triangular meshes, and it can be easily extended to simplicial meshes in higher dimensions.

\section{References}

1. Carlo Arcelli and Gabriella Sanniti di Baja. Euclidean skeleton via centre-ofmaximal-disc extraction. Image and Vision Computing, 11(3):163 - 173, 1993.

2. Oscar Kin-Chung Au, Chiew-Lan Tai, Hung-Kuo Chu, Daniel Cohen-Or, and TongYee Lee. Skeleton extraction by mesh contraction. ACM Transaction on Graphics, 27(3):1-10, August 2008.

3. Thomas Delame, Jacek Kustra, and Alexandru Telea. Structuring 3d medial skeletons: A comparative study. In Symposium on Vision, Modeling and Visualization, 2016.

4. J. Gall, C. Stoll, E. De Aguiar, C. Theobalt, B. Rosenhahn, and H.P. Seidel. Motion capture using joint skeleton tracking and surface estimation. In IEEE Computer Society Conference on Computer Vision and Pattern Recognition (CVPR'09), pages 1746-1753. IEEE Computer Society, June 2009.

5. Dimitri Kudelski, Jean-Luc Mari, and Sophie Viseur. Extraction of feature lines with connectivity preservation. In Computer Graphics International (CGI'11 electronic proceedings), June 2011.

6. Dimitri Kudelski, Sophie Viseur, and Jean-Luc Mari. Skeleton extraction of vertex sets lying on arbitrary triangulated 3D meshes. In Discrete Geometry for Computer Imagery - 17th IAPR International Conference, DGCI 2013, Seville, Spain, March 20-22, 2013. Proceedings, pages 203-214, 2013.

7. Dimitri Kudelski, Sophie Viseur, Giovanni Scrofani, and Jean-Luc Mari. Feature line extraction on meshes through vertex marking and $2 \mathrm{D}$ topological operators. Int. J. Image Graphics, 11(4):531-548, 2011.

8. T.C. Lee, R.L. Kashyap, and C.N. Chu. Building skeleton models via 3-D medial surface/axis thinning algorithms. Graphical Models and Image Processing, 56(6):462-478, November 1994.

9. Jean-Luc Mari. Surface sketching with a voxel-based skeleton. In 15th IAPR International Conference on Discrete Geometry for Computer Imagery (DGCI'09), volume 5810 of Lecture Notes in Computer Science, pages 325-336. Springer, 2009.

10. Christian Rössl, Leif Kobbelt, and Hans-Peter Seidel. Extraction of feature lines on triangulated surfaces using morphological operators. In AAAI Spring Symposium on Smart Graphics, volume 00-04, pages 71-75, March 2000.

11. Punam K Saha, Gunilla Borgefors, and Gabriella Sanniti di Baja. A survey on skeletonization algorithms and their applications. Pattern Recognition Letters, 76:3-12, 2016.

12. Andrea Tagliasacchi, Thomas Delame, Michela Spagnuolo, Nina Amenta, and Alexandru Telea. 3d skeletons: A state-of-the-art report. In Computer Graphics Forum, volume 35, pages 573-597. Wiley Online Library, 2016.

13. Kai Yu, Jiangqin Wu, and Yueting Zhuang. Skeleton-based recognition of chinese calligraphic character image. In Advances in Multimedia Information Processing (PCM'08), volume 5353 of Lecture Notes in Computer Science, pages 228-237. Springer, 2008.

14. T. Y. Zhang and C. Y. Suen. A fast parallel algorithm for thinning digital patterns. Communications of the ACM, 27(3):236-239, March 1984. 